\title{
Excited charmonium physics from lattice QCD
}

\author{
Jozef J. Dudek \\ Jefferson Laboratory, 12000 Jefferson Avenue, Newport News, VA 23606, USA \\ Department of Physics, Old Dominion University, Norfolk, VA 23529, USA
}

\begin{abstract}
Properties of excited mesons are studied using a lattice QCD simulation of a system comparable to charmonium. We extract a spectrum of states, including those with manifestly exotic quantum numbers. Radiative transition form-factors are also computed, in particular the transition from exotic $\eta_{c 1}$ to $J / \psi \gamma$ which is found to be large on the usual scale of magnetic dipole transitions.
\end{abstract}

Keywords: Charmonium, Lattice QCD, Potential Models, Exotics

PACS: PACS: 12.38.Gc, 13.20.Gd, 13.40.Hq, 12.39.Mk

\section{EXCITED CHARMONIUM}

The advent of high-statistics data sets from the B-factories and CLEO-c has lead to a recent explosion in the number of observed meson states in the charmonium region.

The standard framework in which these states can be considered is that of the heavyquark potential model in which charmonia are treated as being $c \bar{c}$ bound-states with the binding provided by a static potential of presumably gluonic origin, but which is determined phenomenologically [1]. This treatment does not directly include light-quark degrees-of-freedomalthough attempts have been made to include their effect perturbatively in many-body extensions $[1,2]$.

Even without the addition of light quarks there is the possibility of states beyond those described by the potential model. The strongly coupled nature of the gluonic field leads us to expect that there can be states in which the gluonic field itself is excited. This can occur in a way which allows a state to have $J^{P C}$ quantum numbers outside the set allowed to a fermion-antifermion pair, such a state is described as being exotic, examples include $0^{+-}, 1^{-+}, 2^{+-}$. It is also possible to produce states with non-exotic $J^{P C}$ which nevertheless have an excited gluonic field internally. Suggestions have been made that the vector $\left(1^{--}\right)$state, the $Y(4260)$, might have just such a structure.

\section{LATTICE QCD}

Lattice QCD offers a method to approximately compute QCD correlators from which observables can be extracted. The principal approximation is the discretisation of Euclidean space-time on to a finite hypercubic grid. The path integral can then be numerically computed by Monte Carlo sampling of possible configurations of gluonic gauge fields. In the calculations reported herein we study a version of QCD in which there are no light quarks, there being only a single heavy flavour whose mass is tuned to be close to that of the charm quark. Virtual loops of quarks of any flavour are explicitly excluded. 


\section{Spectrum}

One of the simplest observables that we can extract from lattice QCD calculations is the spectrum of mesonic states. Composite QCD operators with mesonic quantum numbers can be easily constructed from local fermion bilinears

$$
\mathscr{O}(\vec{x}, t)=\bar{\psi}(\vec{x}, t) \Gamma f(\overleftrightarrow{D}) \psi(\vec{x}, t)
$$

where $\Gamma$ is a Dirac gamma matrix and $f(\overleftrightarrow{D})$ involves some combination of covariant derivatives[3]. Information about the spectrum can be extracted from two-point functions of these operators

$$
C_{i j}(t)=\left\langle 0\left|\sum_{\vec{x}} \mathscr{O}_{i}(\vec{x}, t) \mathscr{O}_{j}^{\dagger}(\overrightarrow{0}, 0)\right| 0\right\rangle=\sum_{\mathfrak{n}}\left\langle 0\left|\mathscr{O}_{i}(\overrightarrow{0}, 0)\right| \mathfrak{n}\right\rangle\left\langle\mathfrak{n}\left|\mathscr{O}_{j}(\overrightarrow{0}, 0)\right| 0\right\rangle e^{-m_{\mathfrak{n}} t}
$$

where the sum over $\vec{x}$ projects the correlator onto zero three-momentum and $|\mathfrak{n}\rangle$ are eigenstates of QCD having the common quantum numbers of $\mathscr{O}_{i, j}$.

A powerful method to extract information about excited states involved attempting to determine what linear combination of some set of operators $\mathscr{O}_{i=1 \ldots N}$ is optimal for a given eigenstate $n$, i.e. $\Omega^{\mathfrak{n}}=\sum_{i=1}^{N} v_{i}^{\mathfrak{n}} \mathscr{O}_{i}$. The best combination, in a variational sense, is obtained from the eigenvectors of the following linear system

$$
C(t) v^{\mathfrak{n}}=\lambda^{\mathfrak{n}}(t) C\left(t_{0}\right) v^{\mathfrak{n}}
$$

where $C(t)$ is a matrix of correlators with elements $C_{i j}(t)$ and the eigenvalues $\lambda^{\mathfrak{n}}(t) \rightarrow$ $e^{-m_{\mathfrak{n}}\left(t-t_{0}\right)}$.

One problem introduced by the hypercubic discretisation is the loss of full rotational symmetry - meson states are no longer distinguished by an integer spin label, but rather by which irreducible representation of the cubic they lie in. In figure 1 we show the spectrum extracted from correlators formed in definite irreps. The colour coding indicates continuum spin-assignment determined by the method described in [3].

\section{Radiative Transitions}

Radiative transition rates are controlled by vector current matrix elements $\left\langle\mathfrak{m}\left|\bar{\psi} \gamma^{\mu} \psi\right| \mathfrak{n}\right\rangle$ which can be extracted from QCD three-point functions

$$
C_{i \mu j}\left(t_{f}, t, t_{i}\right)=\sum_{\vec{x}} e^{-i \vec{p}_{f} \cdot \vec{x}} \sum_{\vec{y}} e^{-i \vec{q} \cdot \vec{y}}\left\langle 0\left|\mathscr{O}_{i}\left(\vec{x}, t_{f}\right) \cdot \bar{\psi}(\vec{y}, t) \gamma^{\mu} \psi(\vec{y}, t) \cdot \mathscr{O}_{j}^{\dagger}\left(\overrightarrow{0}, t_{i}\right)\right| 0\right\rangle .
$$

Using the eigenvectors from the two-point analysis described above, we can project onto a particular state in the spectrum and extract transition matrix elements involving excited states $[4,5]$. The finite extent of the lattice means that three-momentum is discretised such that only certain kinematics are open to us leading to transition matrix elements measured as a function of photon virtuality $Q^{2}$.

In figure 2 we show results for the magnetic dipole from-factor in transitions between vector states and the lightest pseudoscalar state. 

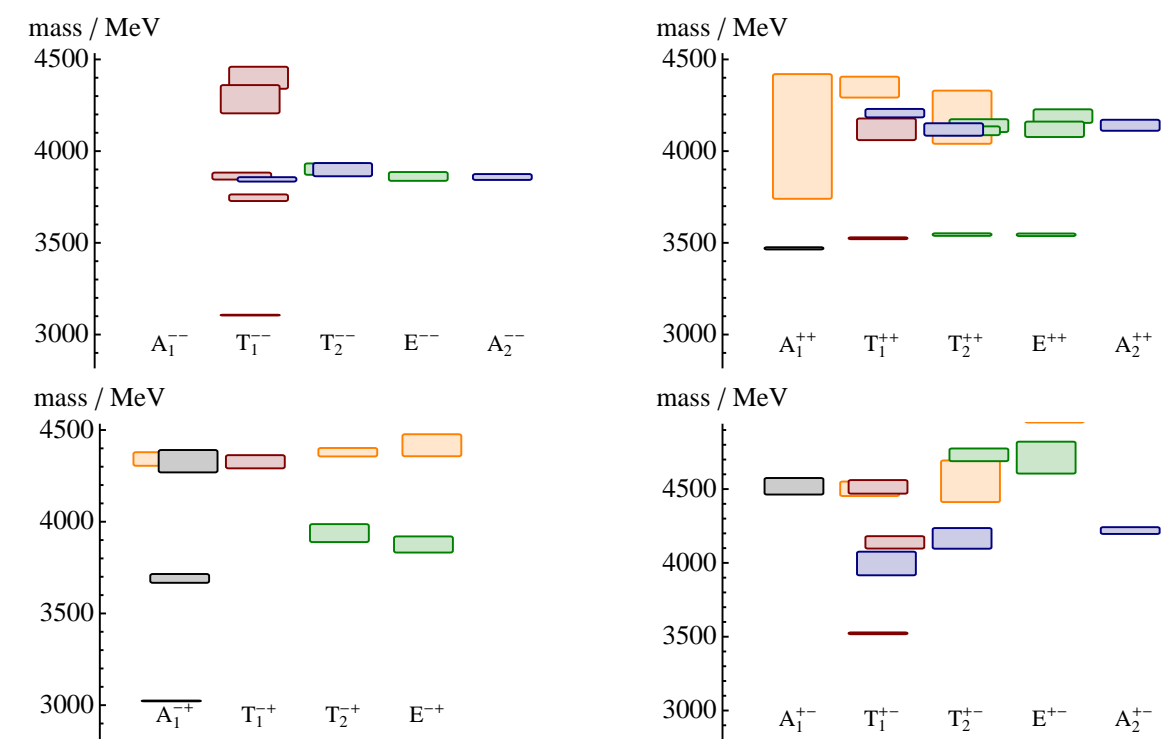

FIGURE 1. Extracted spectrum by lattice irrep $\Lambda^{P C}$. Colour coding indicates continuum spin assignment : $\operatorname{Black}(J=0), \operatorname{Red}(J=1), \operatorname{Green}(J=2)$, Blue $(J=3)$, Orange(unidentified).
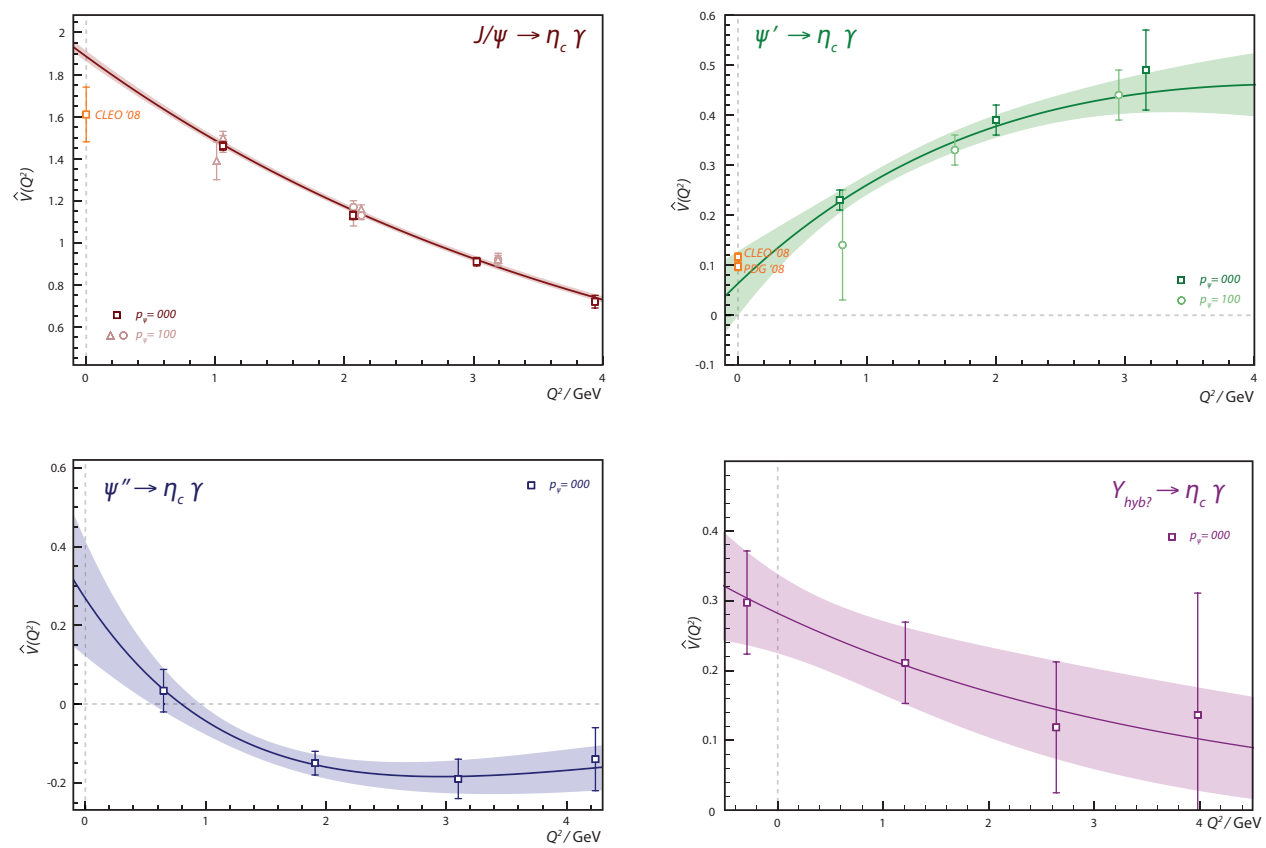

FIGURE 2. Vector-pseudoscalar magnetic dipole transition form-factors. Experimental data at $Q^{2}=0$ from PDG or CLEO-c. Model dependent analysis [6] suggests that the state $Y$ in the fourth pane could be a non-exotic hybrid meson. 


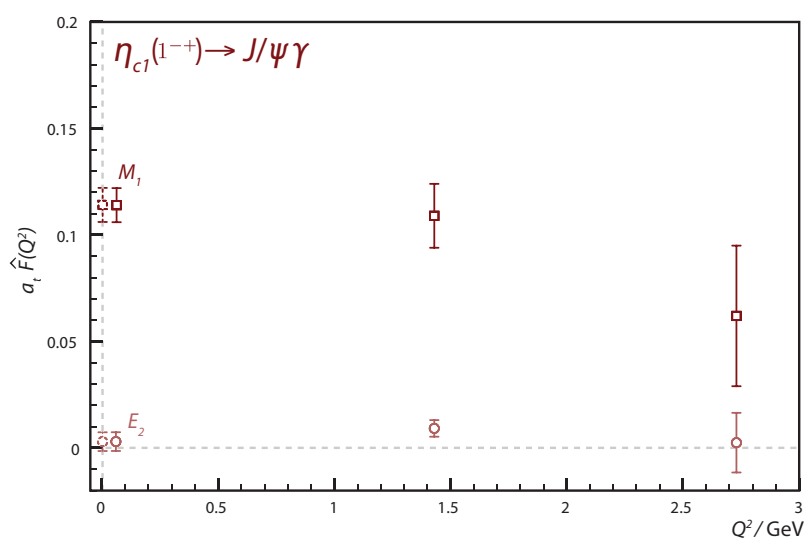

FIGURE 3. Exotic-vector transition form-factors.

Exotic mesons can have radiative transitions to conventional mesons. In figure 3 we show the magnetic dipole and electric quadrupole form-factors for the transition between the $\eta_{c 1}\left(1^{-+}\right)$and the $J / \psi$. The $M_{1}$ piece is clearly non-zero, and compared with the $M_{1}$ amplitude for $J / \psi \rightarrow \eta_{c}$ is very large. A possible explanation for this is that the conventional transition requires a flip of the heavy quark spin, while in the exotic transition it is the excited gluonic field which absorbs the angular momentum. This argument also implies that the exotic $1^{-+}$state has its quarks in a spin-triplet configuration.

\section{FUTURE}

The techniques developed to perform this calculation can also be applied in the light quark sector - the vector current matrix elements there have particular importance in controlling the rate of meson photoproduction at the planned GlueX facility. The large conventional-exotic coupling extracted here, if duplicated in the light quark sector, suggests copious photoproduction of exotic mesons at GlueX. Calculations using distillation technology [7] are underway to determine is this holds true.

\section{ACKNOWLEDGMENTS}

The work presented in this proceedings was performed in collaboration with my esteemed colleagues Robert Edwards, Nilmani Mathur, David Richards, Ermal Rrapaj and Christopher Thomas under the auspices of the Hadron Spectrum Collaboration.

\section{REFERENCES}

1. T. Barnes, S. Godfrey and E. S. Swanson, Phys. Rev. D 72, 054026 (2005)

2. E. Eichten, K. Gottfried, T. Kinoshita, K. D. Lane and T. M. Yan, Phys. Rev. D 17, 3090 (1978)

3. J. J. Dudek, R. G. Edwards, N. Mathur and D. G. Richards, Phys. Rev. D 77, 034501 (2008)

4. J. J. Dudek, R. G. Edwards and D. G. Richards, Phys. Rev. D 73, 074507 (2006)

5. J. J. Dudek, R. G. Edwards and C. E. Thomas, Phys. Rev. D 79, 094504 (2009)

6. J. J. Dudek and E. Rrapaj, Phys. Rev. D 78, 094504 (2008)

7. M. Peardon et al., arXiv:0905.2160 [hep-lat]. 